Commun. Korean Math. Soc. 27 (2012), No. 4, pp. 781-793

http://dx.doi.org/10.4134/CKMS.2012.27.4.781

\title{
SEMI-RIEMANNIAN SUBMANIFOLDS OF A SEMI-RIEMANNIAN MANIFOLD WITH A SEMI-SYMMETRIC NON-METRIC CONNECTION
}

\author{
Ahmet YüCesan And Erol Yaşar
}

\begin{abstract}
We study some properties of a semi-Riemannian submanifold of a semi-Riemannian manifold with a semi-symmetric non-metric connection. Then, we prove that the Ricci tensor of a semi-Riemannian submanifold of a semi-Riemannian space form admitting a semi-symmetric non-metric connection is symmetric but is not parallel. Last, we give the conditions under which a totally umbilical semi-Riemannian submanifold with a semi-symmetric non-metric connection is projectively flat.
\end{abstract}

\section{Introduction}

The notion of a semi-symmetric linear connection on a differentiable manifold was initiated by Friedmann and Schouten [5] in 1924. In 1992, Agashe and Chafle [1] defined a semi-symmetric non-metric connection on a Riemannian manifold and studied the Weyl projective curvature tensor with respect this connection. Moreover, in 1994 they considered in [2] a submanifold admitting a semi-symmetric non-metric connection and studied some of its properties when the ambient manifold is a space form admitting a semi-symmetric nonmetric connection. In 1995, the properties of hypersurfaces of a Riemannian manifold with a semi-symmetric non-metric connection were studied by De and Kamilya [4]. In 2000, Sengupta, De and Binh [9] defined a semi-symmetric non-metric connection which generalized the notion of the semi-symmetric nonmetric connection introduced by Agashe and Chafle. Later, they derived the curvature tensor and the Weyl projective curvature tensor with respect to the semi-symmetric non-metric connection. Prasad and Verma [8], in 2004, got the necessary and sufficient condition in order that the Weyl projective curvature tensor of a semi-symmetric non-metric connection is equal to the Weyl projective curvature of the Riemannian connection. Moreover, they showed that if the curvature tensor with respect to the semi-symmetric non-metric connection

Received August 2, 2011.

2010 Mathematics Subject Classification. 53B15, 53B30, 53C05.

Key words and phrases. semi-symmetric non-metric connection, Levi-Civita connection, semi-Riemannian submanifold, Ricci tensor, projectively flat. 
vanishes, then the Riemannian manifold is projectively flat. Yücesan and Yaşar [11] studied non-degenerate hypersurfaces of a semi-Riemannian manifold with a semi-symmetric non-metric connection and got the conditions under which a non-degenerate hypersurface with a semi-symmetric non-metric connection is projectively flat.

This paper is organized as follows: In Section 2, we consider a semi-Riemannian submanifold immersed in an ambient semi-Riemannian manifold. Then we determine the semi-symmetric non-metric connection, and give the equations of Gauss and Weingarten for a semi-Riemannian submanifold of a semiRiemannian manifold with a semi-symmetric non-metric connection. Furthermore, we show that on a semi-Riemannian submanifold the connection induced from the semi-symmetric non-metric connection is also a semi-symmetric nonmetric connection. In Section 3, by using the equations stated above, we derive Gauss curvature and Codazzi-Mainardi equations with respect to the semisymmetric non-metric connection. In Section 4, we show that the Ricci tensor of a semi-Riemannian submanifold of a semi-Riemannian space form admitting a semi-symmetric non-metric connection is symmetric, but is not parallel. In the last section, we prove that a totally umbilical semi-Riemannian submanifold in a projectively flat semi-Riemannian manifold with a semi-symmetric non-metric connection is projectively flat.

\section{Semi-symmetric non-metric connection}

We suppose that $M$ is an $n$-dimensional semi-Riemannian manifold of an $(n+p)$-dimensional semi-Riemannian manifold $\widetilde{M}$ with semi-Riemannian metric $\widetilde{g}$ of index $0 \leq \nu \leq n+p$. Let us denote by $g$ the induced semi-Riemannian metric tensor on $M$ from $\widetilde{g}$ on $\widetilde{M}$. As $M$ has codimension $p$, we can locally choose $p$ cross sections $\xi_{\alpha}, 1 \leq \alpha \leq p$, of the normal bundle $T M^{\perp}$ of $M$ in $\widetilde{M}$ which are orthonormal at each point of $M$. The index of $\widetilde{g}$ restricted to $T M^{\perp}$ is called the co-index of $M$ in $\widetilde{M}$ and $i n d \widetilde{M}=\nu=i n d M+\operatorname{coind} M$ (see [7]).

A linear connection $\widetilde{\nabla}$ on $\widetilde{M}$ is called a semi-symmetric non-metric connection if its torsion tensor $\widetilde{T}$ satisfies

$$
\widetilde{T}(\widetilde{X}, \tilde{Y})=\widetilde{\pi}(\widetilde{Y}) \widetilde{X}-\widetilde{\pi}(\widetilde{X}) \widetilde{Y}
$$

and

$$
(\widetilde{\nabla} \widetilde{X})(\widetilde{Y}, \widetilde{Z})=-\widetilde{\pi}(\widetilde{Y}) \widetilde{g}(\widetilde{X}, \widetilde{Z})-\widetilde{\pi}(\widetilde{Z}) \widetilde{g}(\widetilde{X}, \widetilde{Y})
$$

for $\widetilde{X}, \widetilde{Y} \in \chi(\widetilde{M})$, where $\widetilde{\pi}$ is a 1 -form on $\widetilde{M}$ (see [1]).

We define a linear connection $\widetilde{\nabla}$ on $\widetilde{M}$ given by

$$
\tilde{\nabla}_{\tilde{X}} \tilde{Y}=\stackrel{\circ}{\widetilde{\nabla}} \widetilde{X} \tilde{Y}+\widetilde{\pi}(\tilde{Y}) \widetilde{X}
$$

for $\tilde{X}, \widetilde{Y} \in \chi(\widetilde{M})$, where $\stackrel{\circ}{\sim}$ denotes the Levi-Civita connection with respect to $\widetilde{g}$ and $\tilde{\pi}$ is a 1 -form associated to a vector field $\widetilde{Q}$ by $\widetilde{g}(\widetilde{Q}, \widetilde{X})=\widetilde{\pi}(\widetilde{X})$ for 
$\widetilde{X} \in \chi(\widetilde{M})$. Then $\widetilde{\nabla}$ is a semi-symmetric non-metric connection on $\widetilde{M}$. On $M$ we define a vector field $Q$ and real valued functions $\mu_{\alpha}, 1 \leq \alpha \leq p$, by decomposing $\widetilde{Q}$ into its unique tangential and normal components, thus

$$
\widetilde{Q}=Q+\sum_{\alpha=1}^{p} \mu_{\alpha} \xi_{\alpha}
$$

If we denote by $\stackrel{\circ}{\nabla}$ the induced Levi-Civita connection on $M$ from $\stackrel{\circ}{\widetilde{\nabla}}$ on $\widetilde{M}$, then we have the Gauss equation with respect to $\stackrel{\circ}{\nabla}$ given by

$$
\stackrel{\circ}{\nabla}_{X} Y=\stackrel{\circ}{\nabla}_{X} Y+\sum_{\alpha=1}^{p} \stackrel{\circ}{h}_{\alpha}(X, Y) \xi_{\alpha}
$$

for $X, Y \in \chi(M)$, where $\stackrel{\circ}{h}_{\alpha}, 1 \leq \alpha \leq p$, are the second fundamental forms on $M$ [7]. Let $\nabla$ on $M$ be induced connection from the semi-symmetric non-metric connection $\widetilde{\nabla}$ on $\widetilde{M}$. Thus, the equation given by

$$
\widetilde{\nabla}_{X} Y=\nabla_{X} Y+\sum_{\alpha=1}^{p} h_{\alpha}(X, Y) \xi_{\alpha},
$$

will be called the Gauss equation with respect to $\widetilde{\nabla}$ for $X, Y \in \chi(M)$, where $h_{\alpha}, 1 \leq \alpha \leq p$, are tensors of type $(0,2)$ on $M$.

Substituting (2.3) and (2.4) into (2.1), we see that

$$
\nabla X Y+\sum_{\alpha=1}^{p} h_{\alpha}(X, Y) \xi_{\alpha}=\stackrel{\circ}{\nabla}_{X} Y+\sum_{\alpha=1}^{p} \stackrel{\circ}{h}_{\alpha}(X, Y) \xi_{\alpha}+\pi(Y) X
$$

from which we get

$$
\nabla_{X} Y=\stackrel{\circ}{\nabla}_{X} Y+\pi(Y) X
$$

where

and we obtain

$$
\pi(Y)=g(Y, Q)
$$

$$
h_{\alpha}=\stackrel{\circ}{h}_{\alpha}, \quad 1 \leq \alpha \leq p,
$$

for $X, Y \in \chi(M)$. By using (2.5), we deduce that

$$
(\nabla \times g)(Y, Z)=-\pi(Y) g(X, Z)-\pi(Z) g(X, Y)
$$

for $X, Y, Z \in \chi(M)$.

Also, from (2.5) the torsion tensor of the connection $\nabla$, denoted by $T$, can be obtained as

$$
T(X, Y)=\pi(Y) X-\pi(X) Y .
$$

Then from (2.7) and (2.8) we have the following theorem: 
Theorem 1. The induced connection on a semi-Riemannian submanifold of a semi-Riemannian manifold with a semi-symmetric non-metric connection is also a semi-symmetric non-metric connection.

The Weingarten equation with respect to $\stackrel{\circ}{\nabla}$ is given by

$$
\stackrel{\circ}{\nabla}_{X} \xi_{\alpha}=-\stackrel{\circ}{A}_{\xi_{\alpha}}(X)+D_{X} \xi_{\alpha}, 1 \leq \alpha \leq p,
$$

for $X \in \chi(M)$, where $D$ is a metric connection on the normal bundle $T M^{\perp}$ with respect to the fibre metric induced from $\widetilde{g}$, and the $(1,1)$ tensor fields $\stackrel{\circ}{A}_{\xi_{\alpha}}, 1 \leq \alpha \leq p$, on $M$ such that

$$
h_{\alpha}(X, Y)=\varepsilon_{\alpha} g\left(\stackrel{\circ}{A}_{\xi_{\alpha}}(X), Y\right)
$$

are called the shape operators of $M \subset \widetilde{M}$ (see [7]).

By virtue of (2.1) and (2.2), we get

$$
\widetilde{\nabla}_{X} \xi_{\alpha}=\stackrel{\circ}{\widetilde{\nabla}_{X}} \xi_{\alpha}+\varepsilon_{\alpha} \mu_{\alpha} X, \quad 1 \leq \alpha \leq p .
$$

From the above and (2.9) it follows that

$$
\tilde{\nabla}_{X} \xi_{\alpha}=-\left(\stackrel{\circ}{A}_{\xi_{\alpha}}-\varepsilon_{\alpha} \mu_{\alpha} I\right)(X)+D_{X} \xi_{\alpha}, 1 \leq \alpha \leq p,
$$

where $I$ is the identity tensor and

$$
\varepsilon_{\alpha}= \begin{cases}-1, & \xi_{\alpha} \text { is timelike } \\ +1, & \xi_{\alpha} \text { is spacelike }\end{cases}
$$

Let the shape operators $A_{\xi_{\alpha}}, 1 \leq \alpha \leq p$, of type $(1,1)$ on $M$ be denoted by

$$
A_{\xi_{\alpha}}=\stackrel{\circ}{A}_{\xi_{\alpha}}-\varepsilon_{\alpha} \mu_{\alpha} I, 1 \leq \alpha \leq p .
$$

So, equation (2.11), called the Weingarten equation with respect to $\tilde{\nabla}$, can be rewritten as

$$
\tilde{\nabla}_{X} \xi_{\alpha}=-A_{\xi_{\alpha}}(X)+D_{X} \xi_{\alpha}, \quad 1 \leq \alpha \leq p
$$

for $X \in \chi(M)$.

By using (2.6), (2.10) and (2.12), we have

$$
\varepsilon_{\alpha} h_{\alpha}(X, Y)=g\left(A_{\xi_{\alpha}} X, Y\right)+\varepsilon_{\alpha} \mu_{\alpha} g(X, Y) .
$$

Let $\xi=\sum_{\alpha=1}^{p} a_{\alpha} \xi_{\alpha}, \eta=\sum_{\alpha=1}^{p} b_{\alpha} \xi_{\alpha}$ be two normal vector fields on $M$. Then from (2.12), we see that

$$
A_{\xi} A_{\eta}=\stackrel{\circ}{A_{\xi}} \stackrel{\circ}{A}_{\eta}-\varepsilon_{\alpha} a_{\alpha} \mu_{\alpha} \stackrel{\circ}{A}_{\eta}-\varepsilon_{\alpha} b_{\alpha} \mu_{\alpha} \stackrel{\circ}{A}_{\xi}+a_{\alpha} b_{\alpha} \mu_{\alpha}^{2} I .
$$

Thus,

$$
\left[A_{\xi}, A_{\eta}\right]=\left[\stackrel{\circ}{A_{\xi}}, \stackrel{\circ}{A}_{\eta}\right]
$$


and

for all $X, Y \in \chi(M)$. Hence we have:

$$
g\left(\left[\stackrel{\circ}{A}_{\xi}, \stackrel{\circ}{A}_{\eta}\right] X, Y\right)=g\left(\left[A_{\xi}, A_{\eta}\right] X, Y\right)
$$

Theorem 2. Let $M$ be a semi-Riemannian submanifold of a semi-Riemannian manifold $\widetilde{M}$ admitting a semi-symmetric non-metric connection. Then the second fundamental tensors with respect to the semi-symmetric non-metric connection are simultaneously diagonalizable if and only if second fundamental tensors with respect to the Levi-Civita connection are simultaneously diagonalizable.

Let $E_{1}, \ldots, E_{\nu}, \ldots, E_{n}$ be the principal vector fields on $M$ corresponding to the unit normal section $\xi=\sum_{\alpha=1}^{p} a_{\alpha} \xi_{\alpha}$ with respect to $\stackrel{\circ}{\sim}$. Then by using (2.12), we have

$$
A_{\xi}\left(E_{i}\right)=\left(\stackrel{\circ}{k}_{i}-\varepsilon_{\alpha} a_{\alpha} \mu_{\alpha}\right) E_{i}, 1 \leq i \leq n
$$

where $\stackrel{\circ}{k}_{i}, 1 \leq i \leq n$, are the principal curvatures corresponding to the unit normal section $\xi$ with respect to the Levi-Civita connection $\stackrel{\circ}{\nabla}$. Taking

$$
k_{i}=\stackrel{\circ}{k}_{i}-\varepsilon_{\alpha} a_{\alpha} \mu_{\alpha}, 1 \leq i \leq n, 1 \leq \alpha \leq p .
$$

So, by (2.16), equation (2.15) is reformed as

$$
A_{\xi}\left(E_{i}\right)=k_{i} E_{i}, 1 \leq i \leq n,
$$

where $k_{i}, 1 \leq i \leq n$, are the principal curvatures of the unit normal section $\xi$ with respect to the semi-symmetric non-metric connection $\tilde{\nabla}$.

From (2.15), (2.16) and (2.17), we assert the following:

Theorem 3. The principal directions of the unit normal direction $\xi$ with respect to the Levi-Civita connection $\stackrel{\circ}{\sim} \underset{\nabla}{ }$ and the semi-symmetric non-metric connection $\widetilde{\nabla}$ coincides and corresponding principal curvatures are equal if and only if $\xi$ is orthogonal to $\widetilde{Q}$.

The mean curvature vector field of $M$ with respect to $\stackrel{\circ}{\nabla}$ is given by

$$
\stackrel{\circ}{H}=\frac{1}{n} \sum_{i=1}^{n} \varepsilon_{i} \sum_{\alpha=1}^{p} \stackrel{\circ}{h}_{\alpha}\left(E_{i}, E_{i}\right) \xi_{\alpha},
$$

where

$$
\varepsilon_{i}= \begin{cases}-1, & E_{i} \text { is timelike } \\ +1, & E_{i} \text { is spacelike }\end{cases}
$$

(see [7]). We define similarly the mean curvature vector field of $M$ with respect to $\nabla$ by

$$
H=\frac{1}{n} \sum_{i=1}^{n} \varepsilon_{i} \sum_{\alpha=1}^{p} h_{\alpha}\left(E_{i}, E_{i}\right) \xi_{\alpha}
$$


From $(2.6),(2.18)$ and $(2.19), H=\stackrel{\circ}{H}$. Hence we have:

Lemma 4. A semi-Riemannian submanifold $M$ of a semi-Riemannian manifold $\widetilde{M}$ admitting a semi-symmetric non-metric connection is totally geodesic with respect to the semi-symmetric non-metric connection if and only if it is totally geodesic with respect to the Levi-Civita connection.

Lemma 5. A semi-Riemannian submanifold $M$ of a semi-Riemannian manifold $\widetilde{M}$ admitting a semi-symmetric non-metric connection is totally umbilical with respect to the semi-symmetric non-metric connection if and only if it is totally umbilical with respect to the Levi-Civita connection.

\section{The Gauss curvature and Codazzi-Mainardi equations}

We denote by

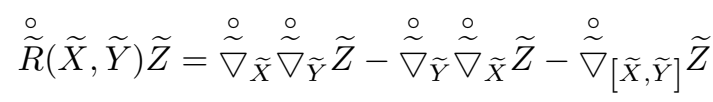

and

$$
\stackrel{\circ}{R}(X, Y) Z=\stackrel{\circ}{\nabla}_{X} \stackrel{\circ}{\nabla}_{Y} Z-\stackrel{\circ}{\nabla}_{Y} \stackrel{\circ}{\nabla}_{X} Z-\stackrel{\circ}{\nabla}_{[X, Y]} Z
$$

the curvature tensors of $\widetilde{M}$ and $M$ with respect to $\stackrel{\circ}{\nabla}$ and $\stackrel{\circ}{\nabla}$, respectively, where $\widetilde{X}, \widetilde{Y}, \widetilde{Z} \in \chi(\widetilde{M})$ and $X, Y, Z \in \chi(M)$. Then the Gauss curvature and Codazzi-Mainardi equations with respect to $\stackrel{\circ}{\sim}$ and $\stackrel{\circ}{\nabla}$, respectively, are given by

$$
\begin{aligned}
\stackrel{\circ}{\widetilde{R}}(X, Y, Z, W)= & \stackrel{\circ}{R}(X, Y, Z, W) \\
& +\sum_{\alpha=1}^{p} \varepsilon_{\alpha}\left\{\stackrel{\circ}{h}_{\alpha}(X, Z) \stackrel{\circ}{h}_{\alpha}(Y, W)-\stackrel{\circ}{h}_{\alpha}(Y, Z) \stackrel{\circ}{h}_{\alpha}(X, W)\right\},
\end{aligned}
$$

and

$$
\begin{aligned}
\stackrel{\circ}{\widetilde{R}}\left(X, Y, Z, \xi_{\alpha}\right)= & \varepsilon_{\alpha}\left\{\left(\stackrel{\circ}{\nabla}_{X} \stackrel{\circ}{h}_{\alpha}\right)(Y, Z)-\left(\stackrel{\circ}{\nabla}_{Y} \stackrel{\circ}{h}_{\alpha}\right)(X, Z)\right\} \\
& +\sum_{\beta=1}^{p} \widetilde{\circ}\left(\stackrel{\circ}{h}_{\beta}(Y, Z) D_{X} \xi_{\beta}-\stackrel{\circ}{h}_{\beta}(X, Z) D_{Y} \xi_{\beta}, \xi_{\alpha}\right)
\end{aligned}
$$

for $X, Y, Z \in \chi(M)$ (see [7]), where

$$
\stackrel{\circ}{\widetilde{R}}(X, Y, Z, W)=\widetilde{g}(\stackrel{\circ}{R}(X, Y) Z, W), \stackrel{\circ}{R}(X, Y, Z, W)=g(\stackrel{\circ}{R}(X, Y) Z, W) .
$$

Now we shall find the Gauss curvature and the Codazzi-Mainardi equations with respect to the semi-symmetric non-metric connections $\tilde{\nabla}$ and $\nabla$. The curvature tensors of $\widetilde{M}$ and $M$ with respect to $\widetilde{\nabla}$ and $\nabla$, respectively, are defined by

$$
\widetilde{R}(X, Y) Z=\tilde{\nabla}_{X} \tilde{\nabla}_{Y} Z-\tilde{\nabla}_{Y} \tilde{\nabla}_{X} Z-\tilde{\nabla}_{[X, Y]} Z
$$


and

$$
R(X, Y) Z=\nabla_{X} \nabla_{Y} Z-\nabla_{Y} \nabla_{X} Z-\nabla_{[X, Y]} Z
$$

for $X, Y, Z \in \chi(M)$.

By using (2.4) and (2.13), we have the curvature tensor of the semi-symmetric non-metric connection $\widetilde{\nabla}$ given by

$$
\begin{aligned}
\widetilde{R}(X, Y) Z= & R(X, Y) Z+\sum_{\alpha=1}^{p}\left\{h_{\alpha}(X, Z) A_{\xi_{\alpha}} Y-h_{\alpha}(Y, Z) A_{\xi_{\alpha}} X\right. \\
& +\left(\nabla_{X} h_{\alpha}\right)(Y, Z) \xi_{\alpha}-\left(\nabla_{Y} h_{\alpha}\right)(X, Z) \xi_{\alpha}+h_{\alpha}(\pi(Y) X-\pi(X) Y, Z) \xi_{\alpha} \\
& \left.+h_{\alpha}(Y, Z) D_{X} \xi_{\alpha}-h_{\alpha}(X, Z) D_{Y} \xi_{\alpha}\right\} .
\end{aligned}
$$

From (3.1), the Gauss curvature equation and the Codazzi-Mainardi equation with respect to $\tilde{\nabla}$ and $\nabla$, respectively, are obtained as:

$$
\begin{aligned}
\widetilde{R}(X, Y, Z, W)= & R(X, Y, Z, W)+\sum_{\alpha=1}^{p} \varepsilon_{\alpha}\left\{h_{\alpha}(X, Z) h_{\alpha}(Y, W)\right. \\
& -h_{\alpha}(Y, Z) h_{\alpha}(X, W)+\mu_{\alpha} h_{\alpha}(Y, Z) g(X, W) \\
& \left.-\mu_{\alpha} h_{\alpha}(X, Z) g(Y, W)\right\}
\end{aligned}
$$

and

$$
\begin{aligned}
\widetilde{R}\left(X, Y, Z, \xi_{\alpha}\right)= & \varepsilon_{\alpha}\left\{\left(\nabla X h_{\alpha}\right)(Y, Z)-\left(\nabla_{Y} h_{\alpha}\right)(X, Z)\right. \\
& \left.+h_{\alpha}(\pi(Y) X-\pi(X) Y, Z)\right\}+\sum_{\beta=1}^{p} \widetilde{g}\left(h_{\beta}(Y, Z) D_{X} \xi_{\beta}\right. \\
& \left.-h_{\beta}(X, Z) D_{Y} \xi_{\beta}, \xi_{\alpha}\right)
\end{aligned}
$$

for $X, Y, Z \in \chi(M)$.

From (2.14) and (3.2), we get

$$
\begin{aligned}
\widetilde{R}(X, Y, X, Y)= & R(X, Y, X, Y)+\sum_{\alpha=1}^{p}\left\{\varepsilon_{\alpha} g\left(A_{\xi_{\alpha}}(X), X\right) g\left(A_{\xi_{\alpha}}(Y), Y\right)\right. \\
& \left.-g\left(A_{\xi_{\alpha}}(X), Y\right)^{2}\right\}+\sum_{\alpha=1}^{p}\left\{\mu_{\alpha} g\left(A_{\xi_{\alpha}}(Y), Y\right) g(X, X)\right. \\
& \left.-\mu_{\alpha} g\left(A_{\xi_{\alpha}}(X), Y\right) g(X, Y)\right\}
\end{aligned}
$$

for $X, Y \in \chi(M)$. Therefore we have the following theorem:

Theorem 6. Let $\mathcal{P}$ be a 2-dimensional non-degenerate subspace of $T_{x} M$, and let $\widetilde{K}(\mathcal{P})$ and $K(\mathcal{P})$ be the sectional curvatures of $\mathcal{P}$ in $\widetilde{M}$ and $M$ with respect to the semi-symmetric non-metric connections $\widetilde{\nabla}$ and $\nabla$, respectively. If $X$ 
and $Y$ form an orthonormal base of $\mathcal{P}$, then

$$
\begin{aligned}
\widetilde{K}(\mathcal{P})= & K(\mathcal{P})+\frac{1}{g(X, X) g(Y, Y)} \sum_{\alpha=1}^{p}\left\{g\left(A_{\xi_{\alpha}}(X), X\right) g\left(A_{\xi_{\alpha}}(Y), Y\right)\right. \\
& \left.-g\left(A_{\xi_{\alpha}}(X), Y\right)^{2}+\mu_{\alpha} g\left(A_{\xi_{\alpha}}(Y), Y\right) g(X, X)\right\}
\end{aligned}
$$

As an immediate consequences of Theorem 6 we obtain:

Corollary 7. If $\widetilde{M}$ is a 3-dimensional flat Lorentz manifold and $M$ is a spacelike or timelike surface in $\widetilde{M}$, then there exists a semi-symmetric non-metric connection $\nabla$ on $M$ for which $\operatorname{det} A_{\xi}$ is an intrinsic invariant of $M$, and when $\widetilde{Q}$ is tangent to $M$, $\operatorname{det} A_{\xi}(=K(\mathcal{P}))$ is equal to $\operatorname{det} \stackrel{\circ}{A}_{\xi}$ which is the Gauss curvature of $M$.

\section{The equation of Ricci with respect to a semi-symmetric non-metric connection}

Let $\xi$ be a normal vector field on $M$. We get

$$
\widetilde{R}(X, Y) \xi=\tilde{\nabla}_{X} \tilde{\nabla}_{Y} \xi-\tilde{\nabla}_{Y} \tilde{\nabla}_{X} \xi-\tilde{\nabla}_{[X, Y]} \xi
$$

where $X, Y \in \chi(M)$. Using (2.12) and (4.1), we have

$$
\begin{aligned}
\widetilde{R}(X, Y) \xi= & R^{N}(X, Y) \xi+\sum_{\alpha=1}^{p}\left\{h_{\alpha}\left(A_{\xi} X, Y\right)-h_{\alpha}\left(A_{\xi} Y, X\right)\right\} \xi_{\alpha} \\
& +A_{D_{X} \xi} Y-A_{D_{Y} \xi} X-\operatorname{Tor}_{A_{\xi}}(X, Y)
\end{aligned}
$$

where $R^{N}$ is the curvature tensor of the normal connection. Using (2.13) and (4.2), we obtain

$$
\widetilde{R}(X, Y, \xi, \eta)=R^{N}(X, Y, \xi, \eta)-g\left(\left[A_{\xi}, A_{\eta}\right] X, Y\right),
$$

where $\eta$ is a normal vector field on $M$. Equation (4.3) is called the equation of Ricci with respect to the semi-symmetric non-metric connection $\widetilde{\nabla}$.

A relation between the curvature tensor of the semi-symmetric non-metric connection $\widetilde{\nabla}$ and the Levi-Civita connection $\stackrel{\circ}{\widetilde{\nabla}}$ is given by

$$
\widetilde{R}(\widetilde{X}, \widetilde{Y}) \widetilde{Z}=\stackrel{\circ}{\widetilde{R}}(\widetilde{X}, \widetilde{Y}) \widetilde{Z}+\widetilde{\alpha}(\widetilde{X}, \widetilde{Z}) \widetilde{Y}-\widetilde{\alpha}(\widetilde{Y}, \widetilde{Z}) \widetilde{X}
$$

where $\widetilde{\alpha}$ is a tensor of type $(0,2)$ defined by

$$
\begin{aligned}
\widetilde{\alpha}(\widetilde{X}, \widetilde{Y}) & =\left(\stackrel{\circ}{\nabla}_{\tilde{X}} \tilde{\pi}\right) \widetilde{Y}-\widetilde{\pi}(\widetilde{X}) \widetilde{\pi}(\widetilde{Y}) \\
& =\left(\widetilde{\nabla}_{\tilde{X}} \widetilde{\pi}\right) \widetilde{Y}
\end{aligned}
$$

for any vector fields $\widetilde{X}, \widetilde{Y}, \widetilde{Z}$ on $\widetilde{M}$. 
Presently, we consider the semi-Riemannian manifold $\widetilde{M}$ with constant curvature $k$. Then we have (see [7])

$$
\stackrel{\circ}{\widetilde{R}}(\widetilde{X}, \widetilde{Y}) \widetilde{Z}=k\{\widetilde{g}(\widetilde{Y}, \widetilde{Z}) \widetilde{X}-\widetilde{g}(\widetilde{X}, \widetilde{Z}) \widetilde{Y}\} .
$$

From (4.4), (4.5) and (4.6), we have

$$
\widetilde{R}(X, Y) \xi=\left(\left(\widetilde{\nabla}_{X} \widetilde{\pi}\right) \xi\right) Y-\left(\left(\widetilde{\nabla}_{Y} \widetilde{\pi}\right) \xi\right) X
$$

for any vector fields $X, Y$ and a normal vector field $\xi$ on $M$. Thus, $\widetilde{R}(X, Y) \xi$ is tangent to $M$ and hence equation (4.3) reduces to

$$
R^{N}(X, Y, \xi, \eta)=g\left(\left[A_{\xi}, A_{\eta}\right] X, Y\right) .
$$

The normal connection $D$ in the normal bundle $T M^{\perp}$ is said to be flat if

$$
R^{N}(X, Y)=D_{X} D_{Y}-D_{Y} D_{X}-D_{[X, Y]}
$$

vanishes identically on $M$.

Hence we have:

Corollary 8. Let $M$ be a semi-Riemannian submanifold of a semi-Riemannian manifold $\widetilde{M}$ with constant curvature admitting a semi-symmetric non-metric connection. Then the normal connection $D$ in the normal bundle $T M^{\perp}$ is flat if and only if all the second fundamental tensors with respect to the semisymmetric non-metric connection are simultaneously diagonalizable.

Theorem 9. The Ricci tensor of a semi-Riemannian submanifold $M$ with respect to the semi-symmetric non-metric connection is symmetric if and only if $\pi$ is closed.

Proof. The Ricci tensor of a semi-Riemannian submanifold $M$ with respect to the semi-symmetric non-metric connection is given by

$$
\operatorname{Ric}(X, Y)=\sum_{i=1}^{n} \varepsilon_{i} g\left(R\left(E_{i}, X\right) Y, E_{i}\right)
$$

for $\forall X, Y \in \chi(M)$. Then using (4.4) in (4.7), we obtain

$$
\operatorname{Ric}(X, Y)=\stackrel{\circ}{\operatorname{Ric}}(X, Y)-(n-1) \alpha(X, Y),
$$

where Ric denotes the Ricci tensor of $M$ with respect to the Levi-Civita connection and

$$
\alpha(X, Y)=\left(\nabla_{X} \pi\right) Y .
$$

From above it follows that

$$
\begin{aligned}
\operatorname{Ric}(X, Y)-\operatorname{Ric}(Y, X) & =(n-1)(\alpha(Y, X)-\alpha(X, Y)) \\
& =2(n-1) d \pi(Y, X)
\end{aligned}
$$

which completes the proof. 
Theorem 10. Let $M$ be a semi-Riemannian submanifold of a semi-Riemannian manifold $\widetilde{M}$. If $\widetilde{R i c}$ and Ric are the Ricci tensor of $\widetilde{M}$ and $M$ with respect to the semi-symmetric non-metric connection, respectively, then for $\forall X, Y \in$ $\chi(M)$

$$
\begin{aligned}
\widetilde{\operatorname{Ric}}(X, Y)= & \operatorname{Ric}(X, Y)-\sum_{\alpha=1}^{p} \varepsilon_{\alpha} f_{\alpha} h_{\alpha}(X, Y)+h_{\alpha}\left(A_{\xi_{\alpha}} X, Y\right) \\
& +n \varepsilon_{\alpha} \mu_{\alpha} h_{\alpha}(X, Y)+\varepsilon_{\alpha} \widetilde{g}\left(\widetilde{R}\left(\xi_{\alpha}, X\right) Y, \xi_{\alpha}\right),
\end{aligned}
$$

where if $\xi_{\alpha}$ is spacelike, $\varepsilon=+1$ or if $\xi_{\alpha}$ is timelike, $\varepsilon=-1$ and $f_{\alpha}=$ $\sum_{i=1}^{n} \varepsilon_{i} h_{\alpha}\left(E_{i}, E_{i}\right)$.

Proof. Let $\left\{E_{1}, \ldots, E_{\nu}, E_{\nu+1}, \ldots, E_{n}, \xi_{1}, \ldots, \xi_{p}\right\}$ be an orthonormal basis of $\chi(\widetilde{M})$. Then the Ricci curvature of $\widetilde{M}$ with respect to the semi-symmetric non-metric connection is given by

$$
\widetilde{\operatorname{Ric}}(X, Y)=\sum_{i=1}^{n} \varepsilon_{i} \widetilde{g}\left(\widetilde{R}\left(E_{i}, X\right) Y, E_{i}\right)+\sum_{\alpha=1}^{p} \varepsilon_{\alpha} \widetilde{g}\left(\widetilde{R}\left(\xi_{\alpha}, X\right) Y, \xi_{\alpha}\right)
$$

for $\forall X, Y \in \chi(M)$. By taking account of (4.9), (3.2), (2.14) and considering the symmetry of shape operators we get (4.8).

Theorem 11. Let $M$ be a semi-Riemannian submanifold of a semi-Riemannian manifold $\widetilde{M}$. If $\widetilde{\rho}$ and $\rho$ are the scalar curvatures of $\widetilde{M}$ and $M$ with respect to the semi-symmetric non-metric connection, respectively, then

$$
\widetilde{\rho}=\rho-\sum_{\alpha=1}^{p} \varepsilon_{\alpha} f_{\alpha}^{2}+n \varepsilon_{\alpha} \mu_{\alpha} f_{\alpha}+f_{\alpha}^{*}+2 \varepsilon_{\alpha} \widetilde{\operatorname{Ric}}\left(\xi_{\alpha}, \xi_{\alpha}\right),
$$

where $f_{\alpha}^{*}=\sum_{i=1}^{n} \varepsilon_{i} h_{\alpha}\left(A_{\xi_{\alpha}} E_{i}, E_{i}\right)$.

Proof. Assume that $\left\{E_{1}, \ldots, E_{\nu}, E_{\nu+1}, \ldots, E_{n}, \xi_{1}, \ldots, \xi_{p}\right\}$ is an orthonormal basis of $\chi(\widetilde{M})$, then the scalar curvature of $\widetilde{M}$ with respect to the semisymmetric non-metric connection is

$$
\widetilde{\rho}=\sum_{i=1}^{n} \varepsilon_{i} \widetilde{\operatorname{Ric}}\left(E_{i}, E_{i}\right)+\sum_{\alpha=1}^{p} \varepsilon_{\alpha} \widetilde{\operatorname{Ric}}\left(\xi_{\alpha}, \xi_{\alpha}\right) .
$$

By virtue of (4.8), (4.11), we obtain (4.10).

We now assume that the 1 -form $\pi$ is closed. Then we can define the sectional curvature for a section with respect to the semi-symmetric non-metric connection (see [1]).

Suppose that the semi-symmetric non-metric connection $\widetilde{\nabla}$ is of constant sectional curvature, then $\widetilde{R}(X, Y) Z$ should be of the form

$$
\widetilde{R}(X, Y) Z=c\{\widetilde{g}(Y, Z) X-\widetilde{g}(X, Z) Y\}
$$


$c$ being a certain scalar. Thus $\widetilde{M}$ is a semi-Riemannian manifold of constant curvature $c$ with respect to semi-symmetric non-metric connection and denote it by $\widetilde{M}(c)$.

Theorem 12. Let $M$ be a semi-Riemannian submanifold of a semi-Riemannian space form $\widetilde{M}(c)$ with a semi-symmetric non-metric connection. Then we have

$$
\begin{aligned}
\operatorname{Ric}(X, Y)= & c(n-1) g(X, Y)+\sum_{\alpha=1}^{p}\left\{\varepsilon_{\alpha} f_{\alpha} h_{\alpha}(X, Y)\right. \\
& \left.-h_{\alpha}\left(A_{\xi_{\alpha}} X, Y\right)-\varepsilon_{\alpha} n \mu_{\alpha} h_{\alpha}(X, Y)\right\}
\end{aligned}
$$

for $\forall X, Y \in \chi(M)$, where $\varepsilon_{i}=g\left(E_{i}, E_{i}\right), \varepsilon_{i}=1$, if $E_{i}$ is spacelike or $\varepsilon_{i}=-1$, if $E_{i}$ is timelike, and $f_{\alpha}=\sum_{i=1}^{n} \varepsilon_{i} h_{\alpha}\left(E_{i}, E_{i}\right)$.

Proof. Taking into account of (4.8) and (4.12), we have (4.13).

From (4.13), the following corollary can be stated as:

Corollary 13. Let $M$ be a semi-Riemannian submanifold of a semi-Riemannian space form $\widetilde{M}(c)$ with a semi-symmetric non-metric connection. Then the Ricci tensor of $M$ is symmetric.

Corollary 14. Let $M$ be a semi-Riemannian submanifold of a semi-Riemannian space form $\widetilde{M}(c)$ with a semi-symmetric non-metric connection. Then the Ricci tensor of $M$ is not parallel.

\section{Projective curvature tensor of a semi-Riemannian submanifold with a semi-symmetric non-metric connection}

We denote by

$$
\stackrel{\circ}{\widetilde{P}}(\widetilde{X}, \widetilde{Y}) \widetilde{Z}=\stackrel{\circ}{\widetilde{R}}(\widetilde{X}, \widetilde{Y}) \widetilde{Z}-\frac{1}{n+p-1}\{\stackrel{\circ}{\operatorname{Ric}}(\widetilde{Y}, \widetilde{Z}) \widetilde{X}-\stackrel{\circ}{\operatorname{Ric}}(\widetilde{X}, \widetilde{Z}) \widetilde{Y}\},
$$

the Weyl projective curvature tensor of an $(n+p)$-dimensional semi-Riemannian manifold $\widetilde{M}$ with respect to the Levi-Civita connection $\stackrel{\circ}{\widetilde{\nabla}}$ for $\widetilde{X}, \widetilde{Y}, \widetilde{Z} \in \chi(\widetilde{M})$, where $\stackrel{\circ}{\text { Ric }}$ is Ricci tensor of $\widetilde{M}$ with respect to the Levi-Civita connection $\stackrel{\circ}{\widetilde{\nabla}}$ (see [3] and [10]).

Analogous to this definition, the Weyl projective curvature tensor of $\widetilde{M}$ with respect to the semi-symmetric non-metric connection can be defined as

$$
\widetilde{P}(\tilde{X}, \widetilde{Y}) \widetilde{Z}=\widetilde{R}(\widetilde{X}, \widetilde{Y}) \widetilde{Z}-\frac{1}{n+p-1}\{\widetilde{\operatorname{Ric}}(\widetilde{Y}, \widetilde{Z}) \widetilde{X}-\widetilde{\operatorname{Ric}}(\widetilde{X}, \widetilde{Z}) \widetilde{Y}\}
$$

for any $\widetilde{X}, \widetilde{Y}, \widetilde{Z} \in \chi(\widetilde{M})$, where $\widetilde{R i c}$ is the Ricci tensor $\widetilde{M}$ with respect to the connection $\widetilde{\nabla}$. Thus, from (5.1), the Weyl projective curvature tensors with 
respect to the semi-symmetric non-metric connection $\widetilde{\nabla}$ and induced connection $\nabla$, respectively, are given by

$$
\begin{aligned}
\widetilde{P}(\widetilde{X}, \widetilde{Y}, \widetilde{Z}, \widetilde{U})= & \widetilde{R}(\widetilde{X}, \widetilde{Y}, \widetilde{Z}, \widetilde{U})-\frac{1}{n+p-1}\{\widetilde{\operatorname{Ric}}(\widetilde{Y}, \widetilde{Z}) \widetilde{g}(\widetilde{X}, \widetilde{U}) \\
& -\widetilde{\operatorname{Ric}}(\widetilde{X}, \widetilde{Z}) \widetilde{g}(\widetilde{Y}, \widetilde{U})\}
\end{aligned}
$$

and

$$
\begin{aligned}
P(X, Y, Z, U)= & R(X, Y, Z, U)-\frac{1}{n-1}\{\operatorname{Ric}(Y, Z) g(X, U) \\
& -\operatorname{Ric}(X, Z) g(Y, U)\}
\end{aligned}
$$

for $\forall X, Y, Z \in \chi(M)$, where

$$
\widetilde{P}(\widetilde{X}, \widetilde{Y}, \widetilde{Z}, \widetilde{U})=\widetilde{g}(\widetilde{P}(\widetilde{X}, \widetilde{Y}) \widetilde{Z}, \widetilde{U}), P(X, Y, Z, U)=g(P(X, Y) Z, U)
$$

and Ric is the Ricci tensor of $M$ with respect to induced connection $\nabla$.

From (5.2), we obtain

$$
\widetilde{P}\left(\xi_{\alpha}, Y, Z, \xi_{\alpha}\right)=\widetilde{R}\left(\xi_{\alpha}, Y, Z, \xi_{\alpha}\right)-\frac{\varepsilon_{\alpha}}{n+p-1} \widetilde{\operatorname{Ric}}(Y, Z) .
$$

Applying (4.8) to (5.4), we have

$$
\begin{aligned}
\operatorname{Ric}(Y, Z)= & \frac{n+p-2}{n+p-1} \widetilde{\operatorname{Ric}}(Y, Z)-\sum_{\alpha=1}^{p}\left\{\varepsilon_{\alpha} \widetilde{P}\left(\xi_{\alpha}, Y, Z, \xi_{\alpha}\right)\right. \\
& \left.+f_{\alpha} \varepsilon_{\alpha} h_{\alpha}(Y, Z)-n \mu_{\alpha} \varepsilon_{\alpha} h_{\alpha}(Y, Z)-h_{\alpha}\left(A_{\xi_{\alpha}} Y, Z\right)\right\} .
\end{aligned}
$$

Then, using (5.2), (5.5) and (3.2) into (5.3) we obtain

$$
\begin{aligned}
& P(X, Y, Z, U) \\
= & \widetilde{P}(X, Y, Z, U)-\sum_{\alpha=1}^{p} \varepsilon_{\alpha}\left\{h_{\alpha}(X, Z) h_{\alpha}(Y, U)-h_{\alpha}(Y, Z) h_{\alpha}(X, U)\right. \\
& \left.+\mu_{\alpha} h_{\alpha}(Y, Z) g(X, U)-\mu_{\alpha} h_{\alpha}(X, Z) g(Y, U)\right\} \\
& +\frac{1}{n-1} \sum_{\alpha=1}^{p} \varepsilon_{\alpha}\left\{\widetilde{P}\left(\xi_{\alpha}, Y, Z, \xi_{\alpha}\right) g(X, U)-\widetilde{P}\left(\xi_{\alpha}, X, Z, \xi_{\alpha}\right) g(Y, U)\right\} \\
& +\frac{p-1}{(n-1)(n+p-1)}(\widetilde{\operatorname{Ric}}(X, Z)-\widetilde{\operatorname{Ric}}(Y, Z)) \\
& +\frac{1}{n-1} g(Y, U)\left\{\sum_{\alpha=1}^{p} \varepsilon_{\alpha} f_{\alpha} h_{\alpha}(X, Z)-n \varepsilon_{\alpha} \mu_{\alpha} h_{\alpha}(X, Z)-h_{\alpha}\left(A_{\xi_{\alpha}} X, Z\right)\right\} \\
& -\frac{1}{n-1} g(X, U)\left\{\sum_{\alpha=1}^{p} \varepsilon_{\alpha} f_{\alpha} h_{\alpha}(Y, Z)-n \varepsilon_{\alpha} \mu_{\alpha} h_{\alpha}(Y, Z)-h_{\alpha}\left(A_{\xi_{\alpha}} Y, Z\right)\right\} .
\end{aligned}
$$

From (5.6), we have the following theorem: 
Theorem 15. A totally umbilical semi-Riemannian submanifold in a projectively flat semi-Riemannian manifold with a semi-symmetric non-metric connection is projectively flat.

\section{References}

[1] N. S. Agashe and M. R. Chafle, A semi-symmetric non-metric connection on a Riemannian manifold, Indian J. Pure Appl. Math. 23 (1992), no. 6, 399-409.

[2] - On submanifolds of a Riemannian manifold with a semi-symmetric non-metric connection, Tensor (N. S.) 55 (1994), no. 2, 120-130.

[3] B. Y. Chen, Geometry of Submanifolds, Marcel-Dekker Inc., New York, 1973.

[4] U. C. De and D. Kamilya, Hypersurfaces of a Riemannian manifold with semisymmetric non-metric connection, J. Indian Inst. Sci. 75 (1995), no. 6, 707-710.

[5] A. Friedmann and J. A. Schouten, Über die Geometrie der halbsymmetrischen Übertragungen, Math. Z. 21 (1924), no. 1, 211-223.

[6] H. A. Hayden, Subspaces of a space with torsion, Proc. London Math. Soc. II. Ser. 34 (1932), 27-50.

[7] B. O'Neill, Semi-Riemannian Geometry with Applications to Relativity, Academic Press, New York, 1983.

[8] B. Prasad and R. K. Verma, On a type of semi-symmetric non-metric connection on a Riemannian manifold, Bull. Calcutta Math. Soc. 96 (2004), no. 6, 483-488.

[9] J. Sengupta, U. C. De, and T. Q. Binh, On a type of semi-symmetric non-metric connection on a Riemannian manifold, Indian J. Pure Appl. Math. 31 (2000), no. 12, 1659-1670.

[10] K. Yano and M. Kon, Structures on Manifolds, World Scientific Co. Pte. Ltd., Singapore, 1984.

[11] A. Yücesan and E. Yaşar, Non-degenerate hypersurfaces of a semi-Riemannian manifold with a semi-symmetric non-metric connection, to appear in Mathematical Reports.

AHMET YÜCESAN

Department of Mathematics

Süleyman Demirel University

ISPARTA 32260, TURKEY

E-mail address: ahmetyucesan@sdu.edu.tr

ERol Yaşar

DePartmet of Mathematics

MERSIN UNIVERSITY

Mezitli Mersin 33343, Turkey

E-mail address: yerol@mersin.edu.tr 